\section{Proposal of sampling protocols to verify possible performance objectives for Campylobacter species control in Italian broiler batches}

\author{
Alessandra De Cesare, ${ }^{1}$ Antonio Valero, ${ }^{2}$ \\ Gerardo Manfreda' \\ 'Dipartimento di Scienze e Tecnologie \\ Agro-Alimentari, Alma Mater Studiorum \\ - Università degli Studi di Bologna, \\ Ozzano Emilia, Italy; ${ }^{2}$ Departamento \\ de Bromatología y Tecnología de los \\ Alimentos, Universidad de Córdoba, \\ Córdoba, Spain
}

\section{Abstract}

Campylobacteriosis represents the most important food-borne illness in the EU. Broilers, as well as poultry meat, spread the majority of strains responsible for human cases. The main aims of this study were to suggest an approach for the definition of performance objectives (POs) based on prevalence and concentration of Campylobacter species (spp.) in broiler carcasses; moreover, sampling plans to determine the acceptability of broiler batches at the slaughterhouses in relation to such P0s were formulated. The dataset used in this study was the one regarding Italy composed during the European Food Safety Authority baseline survey which was performed in the EU in 2008. A total of 393 carcasses obtained from 393 different batches collected from 48 Italian slaughterhouses were included in the analysis. Uncertainty in prevalence and concentration of Campylobacter spp. on carcasses was quantified assuming a beta and log normal distribution. Statistical analysis and distribution fitting were performed in ModelRisk v4.3 (Monte Carlo simulation with 10,000 iterations). By taking the $50^{\text {th }}$ percentile of prevalence distribution as safety limit, sampling plans were subsequently calculated basing on the binomial approach. Final values of number of samples were equal to 4 or 5 to test with qualitative analysis. Considering a limit of quantification of 10 colony forming units/g, a higher number of samples (i.e. 10-13) would be necessary to test using enumeration. An increase of the sensibility of the analytical technique should be necessary to achieve realistic and useful sampling plans based on concentration data.

\section{Introduzione}

La riduzione dei casi di campylobacteriosi umana è diventata una priorità dell'Unione Europea, poiché tale zoonosi è aumentata costantemente dal 2006, e nel 2010 il numero di casi confermati è stato pari a 212.064 (EFSA, 2012). La riduzione dei casi di infezione umana si può ottenere commercializzando una proporzione inferiore di carcasse di polli da carne contaminate, oppure riducendo la contaminazione media del patogeno per carcassa. I livelli di prevalenza e concentrazione che si possono considerare appropriati, in termini di riduzione del rischio di campylobacteriosi umane, si possono esprimere come obiettivi di performance (P0s). Tali obiettivi rappresentano valori quantitativi chiari che le industrie avicole possono cercare di raggiungere.

Il principale obiettivo di questo studio era proporre un approccio per la definizione dei POs in funzione del dato di prevalenza e concentrazione di Campylobacter spp. sulle carcasse di broiler prodotte in Italia, ottenuto durante il monitoraggio EFSA effettuato nel 2008. Inoltre, un piano di campionamento per la verifica di tali POs è stato formulato e potrà essere implementato nelle aziende avicole quando i regolamenti comunitari introdurranno dei limiti relativi alla prevalenza e/o concentrazione di Campylobacter spp. nei polli da carne.

\section{Materiali e Metodi}

Il piano di campionamento per Campylobacter spp. nei polli da carne è stato formulato sulla base dei dati italiani raccolti durante il monitoraggio effettuato dall'EFSA nel 2008, ottenuti nell'ambito del progetto EU denominato BASELINE. Un totale di 393 carcasse, appartenenti ad altrettanti gruppi di polli da carne, sono stati prelevate in 48 macelli distribuiti sul territorio nazionale. La prevalenza e la concentrazione di Campylobacter spp. sulle carcasse sono state determinate analizzando $10 \mathrm{~g}$ di pelle raccolti su collo e petto all'uscita del tunnel di raffreddamento. Il tempo intercorso dalla raccolta delle carcasse alla loro analisi variava da meno di 24 a 80 ore.

L'analisi qualitativa di Campylobacter spp. è stata effettuata attraverso metodica ISO 10272-1:2006 (IS0, 2006a), mentre la conta del patogeno con metodica ISO 10272:2-2006 (ISO, 2006b). I dati di prevalenza sulle carcasse sono stati analizzati mediante distribuzione Beta e il criterio di performance (PO) è stato fissato al $50^{\circ}$ percentile di tale distribuzione. Utilizzando l'approccio suggerito da Whiting et $a l$. (2006), il numero di campioni da analizzare per determinare almeno un campione posi-
Correspondence: Alessandra De Cesare, Dipartimento di Scienze e Tecnologie AgroAlimentari, Alma Mater Studiorum - Università degli Studi di Bologna, via del Florio 2, 40064 Ozzano dell'Emilia, Italy.

Tel. +39.051.2097853 - Fax: +39.051 .2097852 .

E-mail: alessandra.decesare@unibo.it

Key words: Campylobacter spp., Broilers, Performance objective, Sampling plan, Risk assessment.

Conflict of interests: the authors declare no potential conflict of interests.

Funding: this study was financed by the EU project 222738 BASELINE (www.baselineeurope.eu).

Received for publication: 15 January 2013

Revision received: 15 March 2013.

Accepted for publication: 15 March 2013.

This work is licensed under a Creative Commons Attribution 3.0 License (by-nc 3.0).

@C Copyright A. De Cesareet al., 2013

Licensee PAGEPress, Italy

Italian Journal of Food Safety 2013; 2:e8

doi:10.4081/ijfs.2013.e8

tivo segue la seguente equazione:

$$
\mathrm{N}_{\mathrm{s}}=\log \left(1-\mathrm{p}_{\mathrm{R}}\right) / \log (1-\mathrm{P})
$$

dove $\mathrm{N}_{\mathrm{s}}$ è il numero di campioni da analizzare, $\mathrm{p}_{\mathrm{R}}$ è la probabilità di rigettare il campione $(95 \%$ $\mathrm{CL}$ ), e $\mathrm{P}$ è la prevalenza attesa, stimata attraverso l'analisi di distribuzione. Per il calcolo di $\mathrm{N}_{\mathrm{s}}$ è stata effettuata una simulazione MonteCarlo nel ModelRisk v4.3 (Vose Consulting, Ghent, Belgium) con 10.000 interazioni.

\section{Risultati}

La prevalenza di Campylobacter spp. sulle carcasse raccolte durante la sorveglianza effettuata dall'EFSA nel 2008 è risultata variabile dal 45,08 al $61,53 \%$, in funzione del tempo intercorso tra la raccolta del campione e la sua analisi. In particolare, per le carcasse analizzate entro 36 ore dalla raccolta la prevalenza media è risultata pari al $45,08 \%$, mentre per quelle analizzate da 36 a 80 ore dalla raccolta tale prevalenza è risultata pari al $61,53 \%$.

Il PO calcolato per le carcasse analizzate entro 36 ore dalla raccolta è risultato presenza del patogeno in meno del $45,12 \%$ di 5 unita campionarie di pelle di collo e petto. Il PO calcolato, invece, per le carcasse analizzate dopo $36-80$ ore dalla raccolta è risultato presenza del patogeno in meno del $61,45 \%$ di 4 unità cam- 
Tabella 1. Numero di campioni da testare mediante conteggio in funzione del limite di determinazione della metodica.

\begin{tabular}{|c|c|c|c|}
\hline Tempo di analisi & $\begin{array}{l}\text { Numero di } \\
\text { campioni }\end{array}$ & $\begin{array}{c}\text { Limite di } \\
\text { determinazione } \\
\text { (Log UFC/g) }\end{array}$ & $\begin{array}{c}\text { Limite di } \\
\text { determinazione } \\
\text { (UFC/g) }\end{array}$ \\
\hline$<24-36$ ore & $\begin{array}{l}13 \\
11 \\
8 \\
7 \\
6 \\
5\end{array}$ & $\begin{array}{c}1 \\
0,9 \\
0,7 \\
0,6 \\
0,5 \\
0,3\end{array}$ & $\begin{array}{c}10 \\
7,9462 \\
5,0118 \\
3,9810 \\
3,1622 \\
1,9952\end{array}$ \\
\hline $36-80$ ore & $\begin{array}{l}10 \\
9 \\
7 \\
6 \\
5 \\
4\end{array}$ & $\begin{array}{c}1 \\
0,9 \\
0,7 \\
0,6 \\
0,5 \\
0,3\end{array}$ & $\begin{array}{c}10 \\
7,9432 \\
5,0118 \\
3,9810 \\
3,1622 \\
1,9952\end{array}$ \\
\hline
\end{tabular}

pionarie di pelle di collo e petto. Il numero di campioni da analizzare per i due gruppi di carcasse è stato stimato mediante distribuzione binomiale.

La concentrazione media sulle carcasse analizzate entro 36 ore dalla raccolta è risultata pari a $0,3872 \mathrm{Log}$ UFC/g mentre per le carcasse analizzate dopo $36-80$ ore dalla raccolta tale concentrazione è risultata pari a 0,3169 Log UFC/g.

Per formulare un piano di campionamento basato sul conteggio, avendo a disposizione una metodica con un limite di determinazione di $10 \mathrm{UFC} / \mathrm{g}$, il numero di campioni da testare per i due gruppi di carcasse sarebbe pari, rispettivamente, a 13 e 11 (Tabella 1). Tuttavia, riducendo il limite di determinazione della metodica il numero di campioni verrebbe ridotto (Tabella 1).

\section{Discussione}

Prevalenza e concentrazione di Campylobacter spp. nei polli di carne variano notevolmente a livello europeo. Gli obiettivi di performance da stimare sulle carcasse a fine

probabile riduzione delle unità campionarie e dei relativi costi.

I dati di prevalenza e concentrazione ottenuti sulle carcasse variano in funzione del tempo intercorso tra la raccolta del campione e la sua analisi. Tale tempo andrebbe quindi specificato nel piano di campionamento per la determinazione di risultati il più possibile omogenei.

La determinazione di obiettivi di performance chiari per Campylobacter spp. sulle carcasse di pollo e la definizione di piani di campionamento per la loro verifica potrebbe consentire ai produttori un maggior controllo del patogeno con una conseguente riduzione dei casi di campylobacterisi umane.

\section{Bibliografia}

refrigerazione, come pure la stringenza dei piani di campionamento per la sua verifica, dovrebbero quindi essere stabiliti a livello nazionale. Questo studio suggerisce possibili obiettivi di performance ed i relativi piani di campionamento per Campylobacter spp. nei polli da carne in Italia. Tali obiettivi sono stati calcolati in funzione dei dati di prevalenza e concentrazione del patogeno sulle carcasse raccolti durante il monitoraggio effettuato dall'EFSA nel 2008. Se i dati di sorveglianza futuri dovessero indicare prevalenze e concentrazioni diverse, sia i criteri di performance che i corrispondenti piani di campionamento andrebbero modificati.

\section{Conclusioni}

I risultati ottenuti mostrano che il criterio di performance può essere basato sia sull'analisi qualitativa che sul conteggio. In questo ultimo caso, il numero di unità campionarie varia in funzione del limite di determinazione della metodica utilizzata. La possibilità di applicare metodiche molecolari più sensibili rispetto alle metodiche colturali porterebbe quindi ad una
EFSA, 2012. The European Union Summary Report on Trends and Sources of Zoonoses, Zoonotic Agents and Foodborne Outbreaks in 2010. EFSA Journal 10:2597. Disponibile al sito: http://www. efsa.europa.eu/en/search.htm?text=EFSA +Journal+2012\%3B+10\%283\%29\%3A2597

ISO, 2006a. Microbiology of food and animal feeding stuffs. Horizontal method for detection and enumeration of Campylobacter spp. Part 1: Detection method. Norma ISO 10272-1:2006. Organizzazione internazionale per la normazione ed., Ginevra, Svizzera.

ISO, 2006b. Microbiology of food and animal feeding stuffs. Horizontal method for detection and enumeration of Campylobacter spp. Part 2: Colony-count technique. Norma ISO/TS 10272-2:2006. Organizzazione internazionale per la normazione ed., Ginevra, Svizzera.

Whiting RC, Rainosek A, Buchanan RL, Miliotis M, LaBarre D, Long W, Ruple A, Schaub S, 2006. Determining the microbiological criteria for lot rejection from the performance objective or food safety objective. Int J Food Microbiol 110:263-7. 